
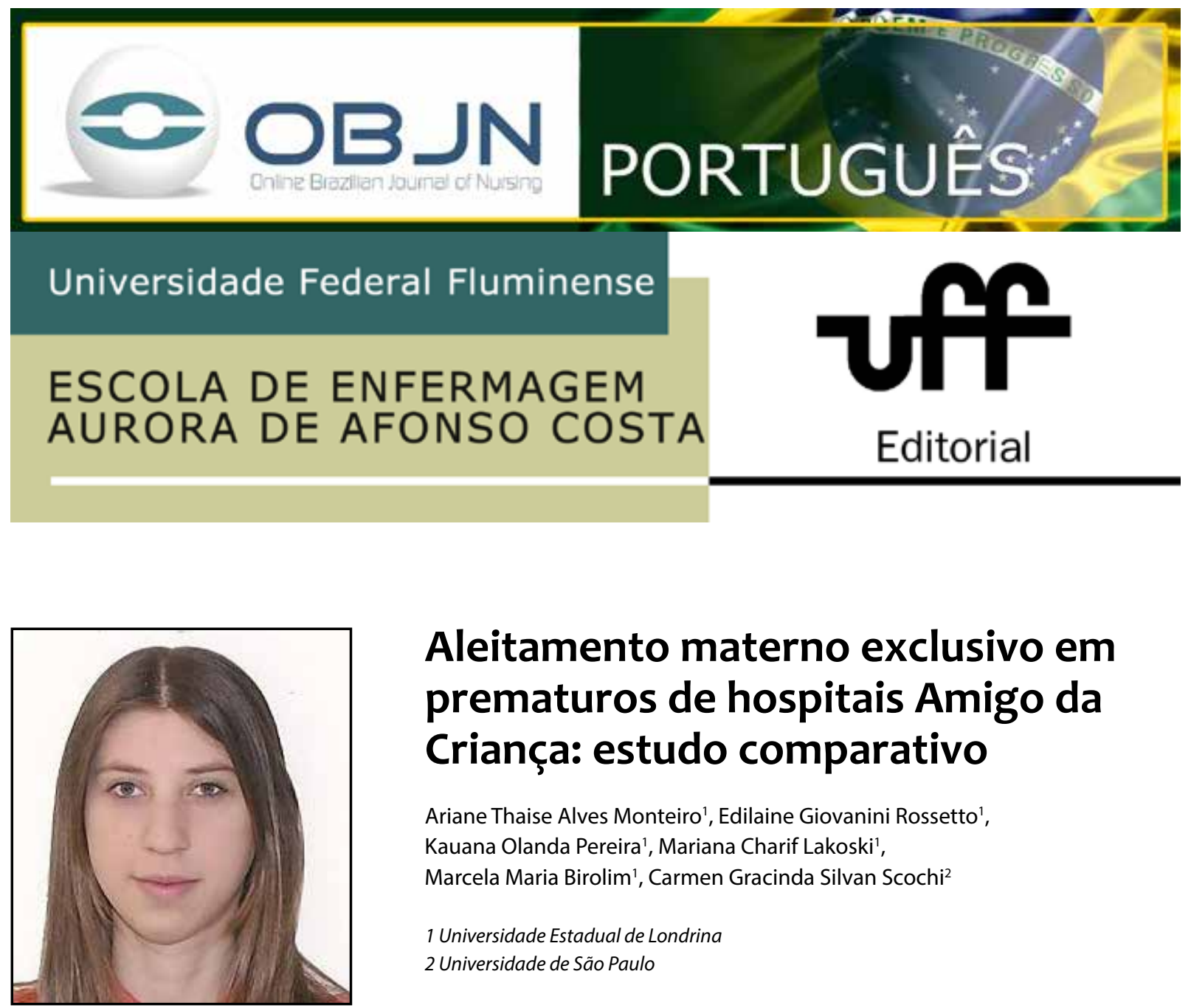

\title{
Aleitamento materno exclusivo em prematuros de hospitais Amigo da Criança: estudo comparativo
}

\author{
Ariane Thaise Alves Monteiro', Edilaine Giovanini Rossetto', \\ Kauana Olanda Pereira', Mariana Charif Lakoski', \\ Marcela Maria Birolim', Carmen Gracinda Silvan Scochi² \\ 1 Universidade Estadual de Londrina \\ 2 Universidade de São Paulo
}

\section{RESUMO}

Objetivo: comparar a prevalência do aleitamento materno exclusivo (AME) e verificar os fatores associados à sua interrupção em prematuros internados em hospitais Amigo da Criança. Método: estudo comparativo com 107 prematuros internados na unidade neonatal de dois hospitais do Paraná. Dados obtidos por meio de entrevistas com as mães e análise de prontuários dos bebês, na alta hospitalar e 15 dias após a mesma. Resultados: as prevalências do AME foram 29,2\% no Hospital Universitário e 15,3\% no Hospital Filantrópico. $\mathrm{Na}$ alta hospitalar, predominou as associações com características clínicas dos bebês, e 15 dias após a alta observou-se maior associação com as características sociodemográficas dos pais. Conclusão: a prevalência de AME, que se mostrou-se baixa no momento da alta hospitalar em ambos os hospitais, reduziu após a alta, reforçando que são necessárias outras estratégias, além daquelas previstas nos hospitais Amigo da Criança, para a promoção e apoio do AME em prematuros.

Palavras-chave: Prevalência; Recém-Nascido Prematuro; Aleitamento Materno; Desmame. 


\section{INTRODUÇÃO}

O leite materno é o alimento ideal para o crescimento e o desenvolvimento do recém-nascido (RN), especialmente em prematuros, por reduzir a mortalidade infantil, prevenir infecções e alergias, além de incentivar o vínculo mãe-bebê ${ }^{(1)}$.

A Organização Mundial da Saúde (OMS) e o Ministério da Saúde preconizam o aleitamento materno exclusivo (AME) até o sexto mês de vida da criança, atribuindo-lhe a seguinte definição: "receber apenas o leite proveniente de sua mãe ou de bancos de leite humano, e nenhum outro líquido ou sólido com exceção de vitaminas, minerais e medicamentos". A definição de aleitamento materno (AM) é classificada quando a criança recebe leite materno (direto da mama ou ordenhado), independentemente de receber ou não outros tipos de alimentos, e o não aleitamento é definido pela criança que não recebe leite materno ${ }^{(2)}$.

Amamentar um pré-termo hospitalizado é um grande desafio, em razão de sua imaturidade fisiológica e neurológica, dificuldade em coordenar a sucção-deglutição-respiração e hospitalização prolongada. Esse contexto gera, na mãe, sentimentos de incapacidade e estresse emocional que podem diminuir a lactação, obsta o contato precoce mãe-filho e promove o início tardio do $\mathrm{AME}$, o que contribui para as baixas taxas de AM nessa clientela ${ }^{(3)}$.

O Brasil é internacionalmente reconhecido pelo desenvolvimento de ações destinadas ao aumento das taxas de $\mathrm{AME}^{(4)}$, como a Iniciativa Hospital Amigo da Criança (IHAC) que foi idealizada em 1990 pela OMS e Fundo das Nações Unidas para a Infância (UNICEF), com o objetivo de promover, proteger e apoiar o AM. Para isso, foi adotada a política dos Dez Passos para o Sucesso do AM em Hospitais Amigos da Criança e sua disseminação tem contribuído expressiva- mente para as taxas de AME e sua duração, com um aumento de $15 \%$ entre 1996 e $2006^{(4)}$.

RN prematuros requerem atenção diferenciada para o apoio à prática do AM. Entretanto, não foram encontrados estudos a respeito da contribuição da IHAC para a garantia do AME durante e após a internação hospitalar, nessa população. Além do mais, o IHAC convencional tem sido apontado como insuficiente para atender essa especificidade ${ }^{(5)}$.

Dessa forma, o objetivo deste estudo foi comparar a prevalência de AME na alta hospitalar e 15 dias após a mesma e verificar os fatores associados à interrupção do AME em bebês prematuros internados em dois hospitais titulados como Amigo da Criança, na região Sul do Brasil.

\section{MÉTODO}

A presente pesquisa faz parte do projeto multicêntrico"Aleitamento materno em prematuros: impacto da IHAC para unidades neonatais", financiado pela Fundação Bill \& Melinda Gates / CNPq. Trata-se de um estudo transversal, comparativo e de abordagem quantitativa.

A pesquisa foi desenvolvida nas unidades neonatais de dois hospitais de grande porte da região Sul do País, sendo um de natureza pública (universitário) e outro filantrópico, que implantaram a estratégia IHAC há pelo menos quinze anos.

Ambas as instituições são consideradas referência regional para gestação de alto risco e atendem grande proporção de prematuros e RN de muito baixo peso. O hospital universitário (HU) atende, exclusivamente, usuários do Sistema Único de Saúde (SUS). O filantrópico (HF), por sua vez, destina $28 \%$ dos seus leitos de maternidade para a clientela SUS e os demais são ocupados por outros serviços complementares, conveniados ou particulares. 
A população do estudo foi constituída por todos os prematuros e suas respectivas mães que obtiveram alta da unidade neonatal no período entre abril e julho de 2014 nos dois hospitais pesquisados. Os critérios de inclusão foram a idade gestacional (IG) menor que 37 semanas e internação na unidade nas primeiras 48 horas de vida, excluindo-se aqueles com contraindicação para AM (ex: malformações da cavidade oral, fenilcetonúria, galactosemia, mãe portadora de HIV, etc.).

A coleta de dados foi realizada em dois momentos: na alta hospitalar do RN e 15 dias após a mesma, esta última realizada via contato telefônico com a mãe, ocasião na qual se empreendeu entrevista sobre a situação do AME / desmame precoce no domicílio.

A variável dependente deste estudo foi a interrupção do AME. As principais variáveis analisadas foram as condições socioeconômicas da família do prematuro, a caracterização da gestação e do parto, a caracterização dos RN prematuros e os fatores relacionados à interrupção do AME na alta hospitalar e no período de 15 dias após à alta.

O programa Statistical Package for the Social Science (SPSS) versão 20.0 foi utilizado para as análises estatísticas. As condições socioeconômicas da família do prematuro, a caracterização da gestação/parto e dos RN prematuros foram analisadas descritivamente para o conhecimento do perfil dos grupos; em seguida foram realizadas, análises de homogeneidade por meio dos testes t-Student, Qui-quadrado e Teste Exato de Fisher. Na sequeência foram obtidas as razões de prevalência (RP) brutas e ajustadas com seus respectivos intervalos de confiança (IC95\%). Variáveis de exposição que, na análise bivariada apresentaram valor de $p<0,20$, foram incorporadas aos modelos multivariados. Utilizou-se o modelo de regressão de Poisson com variância robusta para verificar a existência de fatores as- sociados à interrupção do AME. Permaneceram nos modelos finais as variáveis que mantiveram associação significativa após o ajuste $(p \leq 0,05)$, de acordo com o teste de Wald.

Este estudo foi aprovado pelo Comitê de Ética em Pesquisa da Escola de Enfermagem de Ribeirão Preto da Universidade de São Paulo (EERP/ USP), sob o CAAE no: 23975813.7.1001.5393. A participação na pesquisa foi confirmada por meio da assinatura do Termo de Consentimento Livre e Esclarecido, o qual assegurou o sigilo e anonimato dos participantes.

\section{RESULTADOS}

Foram estudados 107 prematuros e suas mães, sendo 48 da unidade neonatal do $\mathrm{HU}$ e 59 do HF. Observou-se homogeneidade entre os grupos nas seguintes variáveis: idade da mãe, história de parto prematuro, história de amamentação anterior e tipo de gestação. Quanto às demais variáveis, os grupos se mostraram diferentes, visto que apresentaram valor $\mathrm{p} \leq 0,05$ (Tabela 1).

No que se refere às características dos RN prematuros, algumas variáveis mostraram homogeneidade entre os dois grupos, como a IG ao nascer, em que mais da metade dos prematuros nasceram com menos de 34 semanas em ambos os grupos; Apgar, em que a maioria dos RN apresentou valores $\geq 7$ no $5^{\circ}$ minuto; a média de peso e idade gestacional corrigida (IGC) quando mamou pela primeira vez; e a média de peso no momento da alta (Tabela 1).

Cabe ressaltar a caracterização de uma população mais gravemente doente no $\mathrm{HU}$ pelas diferenças observadas entre os grupos no tempo médio de internação, peso ao nascer e Apgar no $5^{\circ}$ minuto de vida, necessidade de nutrição parenteral e tempo de suporte ventilatório/ oxigenioterapia. 
Tabela 1. Caracterização socioeconômica das mães dos prematuros, gestação, parto e dos recém-nascidos prematuros em duas instituições hospitalares. Londrina, PR, Brasil, 2014

\begin{tabular}{|c|c|c|c|}
\hline Características das mães & \multirow{2}{*}{$\frac{\mathbf{H U}(\mathbf{n}=\mathbf{4 8})}{25(15-40)}$} & HF $(n=59)$ & \multirow{3}{*}{$\begin{array}{c}\text { p (Teste) } \\
0,075^{*} \\
0,001^{* *}\end{array}$} \\
\hline Idade da mãe (média, intervalo) & & $27(16-45)$ & \\
\hline Pais residem juntos ( $n, \%)$ & 75,0 & 96,6 & \\
\hline Escolaridade da mãe (n, \%) & & & $0,007 * *$ \\
\hline$<8$ anos de estudo & 22,9 & 5,1 & \\
\hline$\geq 8$ anos de estudo & 77,1 & 94,9 & \\
\hline Trabalha fora de casa $(n, \%)$ & 58,3 & 37,3 & $0,030^{* *}$ \\
\hline Renda mensal familiar (n, \%) & & & $0,000 * *$ \\
\hline 1 a 2 salários mínimos & 64,6 & 6,8 & \\
\hline$\geq 3$ salários mínimos & 35,4 & 93,2 & \\
\hline Local do pré-natal $(n, \%)$ & & & $0,000 * *$ \\
\hline Posto/Unidade de saúde & 100,0 & 8,5 & \\
\hline Outro/Particular & 0,0 & 91,5 & \\
\hline História parto prematuro (n, \%) & 8,3 & 6,8 & $1,000^{* * *}$ \\
\hline Amamentação anterior ( $n, \%)$ & 70,8 & 83,0 & $0,132^{* *}$ \\
\hline Tipo de gestação (n, \%) & & & $0,064 * *$ \\
\hline Única & 79,2 & 62,7 & \\
\hline Dupla ou mais & 20,8 & 37,3 & \\
\hline Intercorrências na gestação (n, \%) & 62,5 & 81,4 & $0,029 * *$ \\
\hline Intercorrências no parto (n, \%) & 27,1 & 1,7 & $0,000^{* *}$ \\
\hline Tipo de parto $(n, \%)$ & & & $0,000 * *$ \\
\hline Vaginal & 39,6 & 6,8 & \\
\hline Cesárea & 60,4 & 93,2 & \\
\hline Características do RN & HU (n=48) & HF $(n=59)$ & \\
\hline Sexo do RN (n, \%) & & & \\
\hline Feminino & 60,4 & 37,3 & $0,017 * *$ \\
\hline Masculino & 39,6 & 62,7 & \\
\hline IG nascimento (n, \%) & & & \\
\hline$<34$ sem & 62,5 & 57,6 & $0,609 * *$ \\
\hline$\geq 34$ sem & 37,5 & 42,4 & \\
\hline Reanimação neonatal (n, \%) & 31,2 & 13,5 & $0,027^{* * *}$ \\
\hline Apgar $5^{\circ}$ minuto $(n, \%)$ & & & \\
\hline$<7$ & 10,4 & 1,7 & $0,088^{* * *}$ \\
\hline$\geq 7$ & 89,6 & 98,3 & \\
\hline Nutrição parenteral (n, \%) & 87,5 & 23,7 & $0,000^{* *}$ \\
\hline Tempo de suporte ventilatório, dias (média, intervalo) & $\begin{array}{c}8 \\
(0-67)\end{array}$ & $\begin{array}{c}4 \\
(0-21)\end{array}$ & $0,033^{*}$ \\
\hline Peso ao nascer, gramas (média, intervalo) & $\begin{array}{c}1741 \\
(765-2670)\end{array}$ & $\begin{array}{c}1943 \\
(1010-2635)\end{array}$ & $0,033^{*}$ \\
\hline $\begin{array}{l}\text { IGC mamou } 1^{\circ} \mathrm{vez} \text {, semanas } \\
\text { (média, intervalo) }\end{array}$ & $\begin{array}{c}35 \\
(32-40)\end{array}$ & $\begin{array}{c}35 \\
(31-52)\end{array}$ & $0,924^{*}$ \\
\hline Peso mamou $1^{\circ}$ vez, gramas (média, intervalo) & $\begin{array}{c}1975 \\
(1475-2704)\end{array}$ & $\begin{array}{c}2029 \\
(1465-2580)\end{array}$ & $0,394^{*}$ \\
\hline $\begin{array}{l}\text { IGC na alta, semanas } \\
\text { (média, intervalo) }\end{array}$ & $\begin{array}{c}37 \\
(34-48)\end{array}$ & $\begin{array}{c}36 \\
(33-39)\end{array}$ & $0,005^{*}$ \\
\hline $\begin{array}{l}\text { Peso na alta, gramas } \\
\text { (média, intervalo) }\end{array}$ & $\begin{array}{c}2145 \\
(1570-3656)\end{array}$ & $\begin{array}{c}2092 \\
(1660-2765)\end{array}$ & $0,354^{*}$ \\
\hline Duração da internação, dias (média, intervalo) & $\begin{array}{c}31 \\
(5-116)\end{array}$ & $\begin{array}{c}18 \\
(2-47)\end{array}$ & $0,003^{*}$ \\
\hline
\end{tabular}

${ }^{*}$ t-Student; ${ }^{* *}$ Qui-quadrado; ${ }^{* * *}$ Teste Exato de Fisher

Fonte: o próprio autor. 
A Figura 1 compara a prevalência do AME dos prematuros no momento da alta hospitalar e após 15 dias. Verificou-se que as prevalências nos dois hospitais tiveram pouca variação, se comparado o momento da alta hospitalar com a primeira quinzena após a alta, e não apresentou diferença estatisticamente significante quando comparadas às prevalências entre os dois hospitais nos dois momentos $(p=0,096$ e $p=0,095)$.

As prevalências de AME dos prematuros nos dois hospitais mostraram-se baixas na alta hospitalar: 29,2\%, no HU; e 15,3\%, no HF.

Figura 1. Prevalência do aleitamento materno exclusivo em prematuros no momento da alta hospitalar e na primeira quinzena após a alta em duas instituições hospitalares. Londrina, PR, Brasil, 2014.

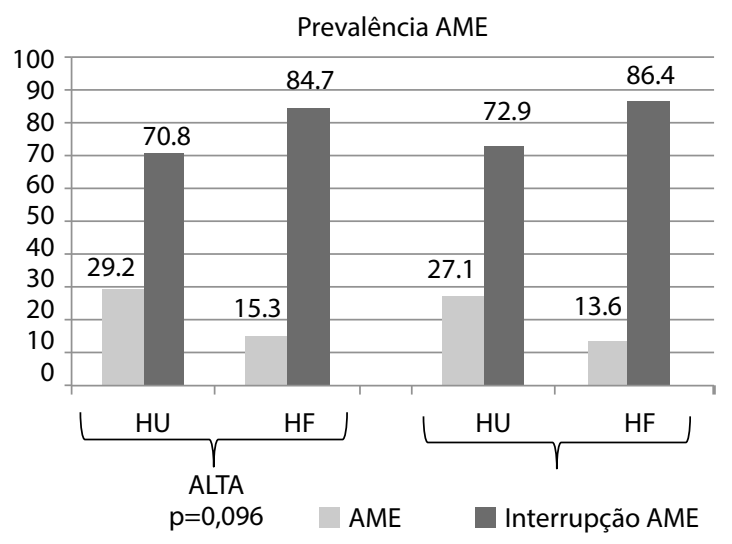

Fonte: o próprio autor.

Ao realizar a análise bivariada, as variáveis que apresentaram valor $p<0,20$ foram incorporadas ao modelo multivariado da Regressão de Poisson e analisadas pela RP ajustada em relação à interrupção ao AME na alta (Tabela 2) e na primeira quinzena após a alta hospitalar (Tabela 3).

A prevalência para a interrupção do AME no HU foi $38 \%$ maior no parto vaginal, ou seja, o parto cesárea foi protetor para o AME desta população, assim como o sexo masculino, dado que o feminino teve uma prevalência $82 \%$ maior para cessar o AME na alta. Para o uso de oxigenoterapia, a prevalência da interrupção do AME foi $41 \%$ maior para os prematuros que fizeram uso de oxigênio (Tabela 2).

No HF, entre os dez fatores restantes do modelo bivariado, seis mostraram associação estatisticamente significante para a descontinuidade do AME na alta, sendo diferentes variáveis se comparadas ao HU: história de parto prematuro, intercorrência no parto, reanimação em sala de parto, Apgar menor que 7 no $5^{\circ}$ minuto e uso de oxigenoterapia.

A prevalência para a interrupção do AME no HU após a alta hospitalar foi $71 \%$ maior nos casos de gemelaridade, $41 \%$ maior em bebês que fizeram uso de oxigênio e $36 \%$ maior em mães que tiveram trauma mamilar (Tabela 3).

No HF, fatores socioeconômicos, como pais não residirem juntos e baixa escolaridade materna, estiveram associados à interrupção do AME (50\% e 57\%) 15 dias após a alta hospitalar. A prevalência do não AME foi $29 \%$ maior para história de parto prematuro, 62\% maior nos bebês que tiveram intercorrência no parto, 74\% maior em bebês que foram reanimados na sala de parto, $41 \%$ para uso de oxigenoterapia, $14 \%$ para trauma mamilar, 39\% para Apgar menor que 7 no $5^{\circ}$ minuto e quanto menor a IG ao nascer, maior foi a prevalência da descontinuidade do AME na primeira quinzena no domicílio.

\section{DISCUSSÃO}

Embora haja estudos publicados sobre a prevalência de $A M$ em prematuros ${ }^{(6-7)}$ e a prevalência de $A M$ em instituições Amiga da Criança ${ }^{(4-5,8)}$, não foi encontrada pesquisa que avaliasse o AM de prematuros em instituições Amiga da Criança, tal como o proposto por este estudo.

Apesar de ambas as instituições estudadas possuírem o título de Hospital Amigo da Criança, ao compará-las se constatou prevalências diferentes clinicamente, com ressalvas para as diferenças encontradas entre os dois grupos. A 
Tabela 2. Fatores associados com a interrupção do AME em prematuros no momento da alta em dois hospitais amigos da criança. Londrina, PR, Brasil, 2014.

\begin{tabular}{|c|c|c|c|c|c|c|}
\hline \multirow{3}{*}{ Fatores } & \multicolumn{6}{|c|}{ Interrupção AME na alta } \\
\hline & \multicolumn{3}{|c|}{ HU } & \multicolumn{3}{|c|}{ HF } \\
\hline & n (\%) & $\begin{array}{c}\text { RP bruta* } \\
\text { IC 95\% }\end{array}$ & $\begin{array}{l}\text { RP ajustado } \\
\text { IC 95\% }\end{array}$ & n (\%) & $\begin{array}{c}\text { RP bruta* } \\
\text { IC 95\% }\end{array}$ & $\begin{array}{c}\text { RP ajustado } \\
\text { IC 95\% }\end{array}$ \\
\hline $\begin{array}{l}\text { Sexo do RN } \\
\text { Feminino } \\
\text { Masculino }\end{array}$ & $\begin{array}{c}25(86,2) \\
9(47,4)\end{array}$ & $\begin{array}{c}1,82 \\
(1,10-1,98)\end{array}$ & $\begin{array}{c}1,82 \\
(1,19-1,79)\end{array}$ & $\begin{array}{l}20(90,9) \\
30(81,1)\end{array}$ & - & - \\
\hline $\begin{array}{l}\text { Reanimação neo- } \\
\text { natal } \\
\text { Sim } \\
\text { Não }\end{array}$ & $\begin{array}{l}11(73,3) \\
23(69,7)\end{array}$ & - & - & $\begin{array}{l}8(100,0) \\
42(82,3)\end{array}$ & $\begin{array}{c}1,21 \\
(1,06-1,37)\end{array}$ & $\begin{array}{c}1,47 \\
(1,14-1,90)\end{array}$ \\
\hline $\begin{array}{l}\text { Apgar } 5^{\circ} \text { minuto } \\
<7 \\
\geq 7\end{array}$ & $\begin{array}{c}3(60,0) \\
31(72,1)\end{array}$ & - & - & $\begin{array}{l}1(100,0) \\
49(84,5)\end{array}$ & $\begin{array}{c}1,18 \\
(1,06-1,32)\end{array}$ & $\begin{array}{c}0,79 \\
(0,66-0,94)\end{array}$ \\
\hline $\begin{array}{l}\text { Uso de } 02 \\
\text { Sim } \\
\text { Não }\end{array}$ & $\begin{array}{l}28(66,7) \\
6(100,0) \\
\end{array}$ & $\begin{array}{c}0,66 \\
(0,53-0,82)\end{array}$ & $\begin{array}{c}0,59 \\
(0,42-0,82)\end{array}$ & $\begin{array}{l}48(84,2) \\
2(100,0)\end{array}$ & $\begin{array}{c}0,84 \\
(0,75-094)\end{array}$ & $\begin{array}{c}0,59 \\
(0,43-0,82)\end{array}$ \\
\hline & & & ÁVEIS MÃE & & & \\
\hline $\begin{array}{l}\text { História parto pre- } \\
\text { maturo } \\
\text { Sim } \\
\text { Não }\end{array}$ & $\begin{array}{l}4(100,0) \\
30(68,1)\end{array}$ & $\begin{array}{c}1,46 \\
(1,19-1,79)\end{array}$ & ** & $\begin{array}{l}4(100,0) \\
46(83,6)\end{array}$ & $\begin{array}{c}1,19 \\
(1,06-1,34)\end{array}$ & $\begin{array}{c}1,42 \\
(1,10-1,84)\end{array}$ \\
\hline $\begin{array}{l}\text { Intercorrências no } \\
\text { parto } \\
\text { Sim } \\
\text { Não }\end{array}$ & $\begin{array}{l}11(84,6) \\
23(65,7)\end{array}$ & $\begin{array}{c}1,28 \\
(0,92-1,79)\end{array}$ & ** & $\begin{array}{l}1(100,0) \\
49(84,5)\end{array}$ & $\begin{array}{c}1,18 \\
(1,06-1,32)\end{array}$ & $\begin{array}{c}0,69 \\
(0,53-0,90)\end{array}$ \\
\hline $\begin{array}{l}\text { Tipo de parto } \\
\text { Vaginal } \\
\text { Cesárea }\end{array}$ & $\begin{array}{l}16(84,2) \\
18(62,1)\end{array}$ & $\begin{array}{c}0,73 \\
(0,52-1,04)\end{array}$ & $\begin{array}{c}0,62 \\
(0,45-0,86)\end{array}$ & $\begin{array}{l}4(100,0) \\
46(83,6)\end{array}$ & $\begin{array}{c}0,83 \\
(0,74-0,94)\end{array}$ & $* *$ \\
\hline $\begin{array}{l}\text { Trauma Mamilar } \\
\text { Sim } \\
\text { Não }\end{array}$ & $\begin{array}{l}01(33,3) \\
33(73,3)\end{array}$ & - & - & $\begin{array}{l}28(93,3) \\
22(75,8)\end{array}$ & $\begin{array}{c}1,23 \\
(0,98-1,54)\end{array}$ & $* *$ \\
\hline
\end{tabular}

* variáveis com $p \leq 0,20$ no modelo bivariado

** variáveis com valor de $p>0,05$ no modelo multivariado.

Fonte: o próprio autor.

população estudada no HF tem melhor condição socioeconômica, visto que é uma instituição que atende, além de gestantes de alto risco do SUS, pacientes conveniadas e particulares. A população do HU apresenta características de nível socioeconômico mais precárias: um quarto das mães não têm parceiros conjugais; quase $30 \%$ possuem menos de oito anos de estudo; mais da metade trabalham fora de casa e apresentaram renda mensal entre um a dois salários mínimos. Não obstante, os prematuros do HU apresentaram maior prevalência de AME na alta e após a alta.

Os pais residirem juntos e a escolaridade materna de mais de 8 anos de estudo se revelaram como fatores de proteção para a continuidade do AME após a alta no HF. Mães que vivem sem companheiro têm mais chance de abandonar precocemente o AME que mães com companheiro ${ }^{(8)}$. O tempo de escolarida- 
Tabela 3. Fatores associados com a interrupção do AME em prematuros na primeira quinzena após a alta em duas instituições hospitalares. Londrina, PR, Brasil, 2014.

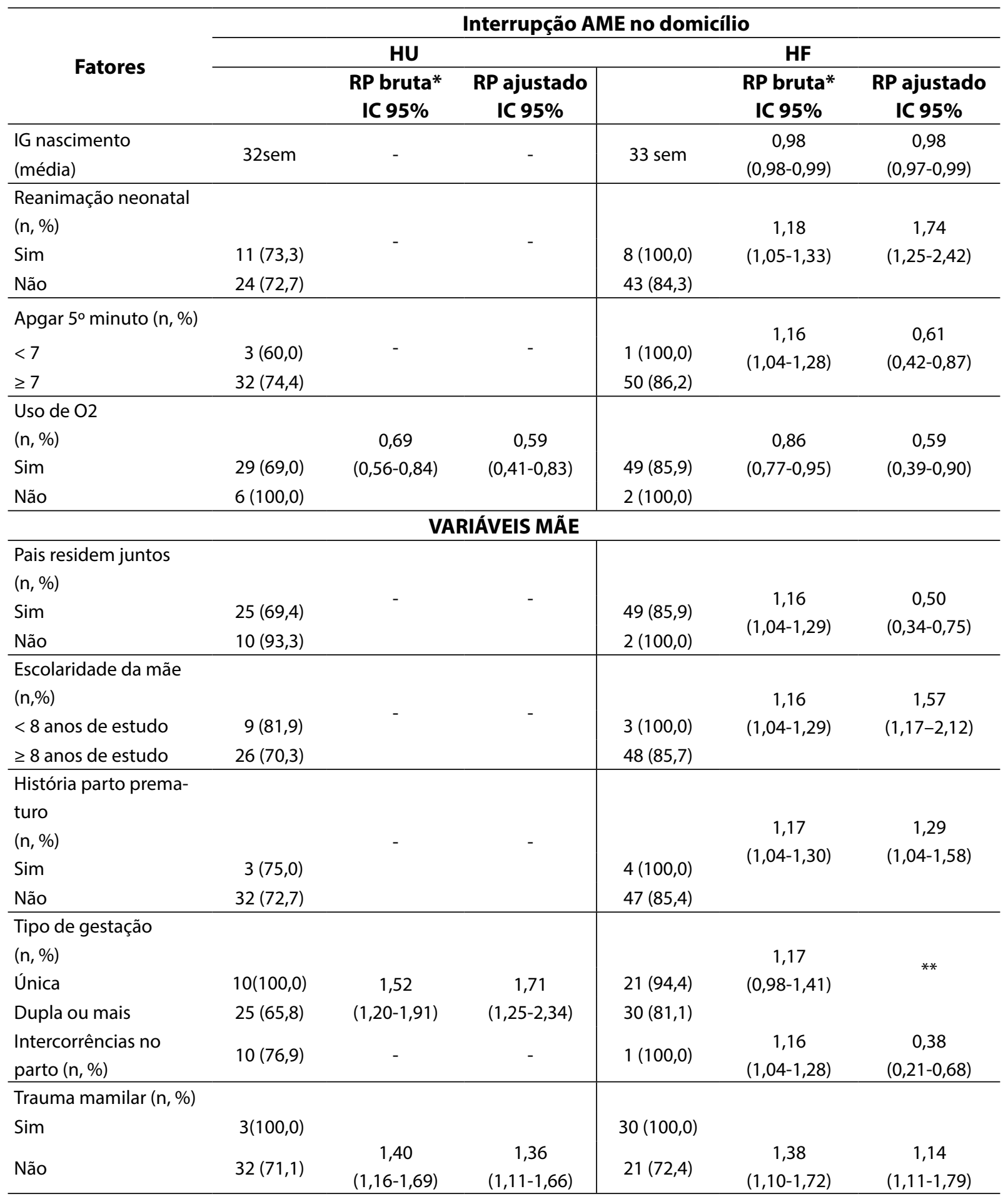

* variáveis com $p \leq 0,20$ no modelo bivariado

** variáveis com valor de $p>0,05$ no modelo multivariado.

Fonte: o próprio autor. 
de da mãe tem sido apontado como um dos indicadores mais importantes para apontar o grau de esclarecimento da mãe, o que reflete na compreensão e acesso sobre os benefícios do $A M$, principalmente sobre os riscos de um nascimento prematuro ${ }^{(8)}$.

A necessidade de oxigenoterapia foi um fator associado com a interrupção do AME, tanto na alta como após a alta hospitalar, no HU e no HF. Entre os fatores associados com a não continuidade do AME comuns ao HF na alta e após a alta estavam: história de parto prematuro anterior, intercorrências no parto, necessidade de reanimação na sala de parto e o Apgar menor que 7 no $5^{\circ}$ minuto. Tais fatores aumentam a gravidade do paciente e podem prejudicar o início e a manutenção do AM do prematuro. Adiando o início precoce do AM, o RN permanece mais tempo internado, separado de sua mãe, o que dificulta ainda mais o estabelecimento da amamentação ${ }^{(8,9)}$.

Cabe ressaltar as diferenças na gravidade dos prematuros do HU. Um terço dos prematuros precisaram de reanimação na sala de parto; necessitaram duas vezes mais dias de uso de suporte ventilatório/oxigenoterapia que os prematuros do HF; permaneceram, em média, o dobro do tempo internados; e a maioria necessitou de nutrição parenteral. Ademais, o peso ao nascer mínimo foi de 765 gramas no HU, enquanto no HF nenhum bebê nasceu pesando menos que 1000 gramas.

Apesar do peso ao nascer não ter apresentado significância estatística com a interrupção do AME nas duas instituições, diferente da IG ao nascer, a qual, no HF, demonstrou relação na primeira quinzena após a alta hospitalar, vale destacar que a prática do AM em prematuros está associada às duas variáveis. A literatura aponta que quanto menor o peso e/ou menor a IG, maior a ocorrência de dificuldade para iniciar e manter a amamentação(9).
Embora nascer menos prematuro seja um fator favorável para o $\mathrm{AME}$, cabe destacar os prematuros denominados tardios, isto é, aqueles que apresentam IG entre 34 e 36 semanas e 6 dias. Esse novo agrupamento baseou-se na necessidade de dar ênfase aos RN próximos da classificação a termo, mas que ainda são prematuros e, portanto, deveriam receber maiores cuidados quando em comparação ao RN a termo(10).

Os prematuros tardios têm sido cada vez mais frequentes e estão significativamente mais associados com o aumento da morbimortalidade neonatal, quando em comparação com RN a termo, que possuem maior risco de associação a intercorrências no período neonatal ${ }^{(10,11)}$. Eles têm sido descritos com menores escores de Apgar, maior risco de problemas respiratórios e alimentares, desidratação, instabilidade térmica, sucção e deglutição deficientes, icterícia e hipoglicemia e são mais suscetíveis à reinternação nas primeiras semanas de vida ${ }^{(11)}$.

Considerando todos esses riscos e intercorrências, os prematuros tardios exigem um conjunto de intervenções para o sucesso do $A M E$, pois apresentam limitações que exigem mais atenção e ajuda para iniciar e estabelecer o AM que o bebê a termo, as quais muitas vezes são negligenciadas pela equipe ${ }^{(11)}$.

No HF, cuja população apresenta melhores condições de vida e possui plano de saúde, o número de partos cesáreas foi expressivo. Independente da indicação precisa do tipo de parto, que não era objeto desta pesquisa, outros estudos apontaram que interromper a gestação antes das 37 semanas completas ocorre mais frequentemente no setor privado, e, além de aumentar os riscos de morbimortalidade desses bebês, também é um fator que dificulta o estabelecimento do $\mathrm{AM}^{(1,12-13)}$. Por outro lado, um estudo aborda que o parto cesárea de emergência, como no caso de um RN prematuro, não apresenta efeito no $\mathrm{AM}^{(14)}$. No presente estudo, 
a cesárea teve fator protetor para o AME em prematuros nascidos no $\mathrm{HU}$, dada a condição de prematuridade extrema.

Curiosa é a associação do sexo com o AM que ainda não conseguiu ser suficientemente explicada. Um estudo dinamarquês com 1.488 prematuros, com IG de 24 a 36 semanas, demonstrou pela análise multivariada que ser menino é um fator que contribuiu para o insucesso do AME na alta ${ }^{(13)}$, diferentemente do presente estudo que encontrou uma associação positiva entre o sexo masculino e AME na alta no HU.

O tempo de internação não mostrou significância estatística como fator associado à descontinuidade do $A M E$, tal como em outro estudo que apontou aumento da chance de se manter o AME conforme diminuição do tempo de internação prolongado(6). O tempo de internação aumenta a separação da mãe com seu filho e o risco do RN adquirir infecções e demais complicações clínicas. Ademais, prejudica a formação do vínculo mãe-bebê, dificultando, assim, o início e a manutenção da amamentação ${ }^{(3,9)}$.

Por outro lado, o tempo de internação prolongado pode acabar influenciando positivamente a amamentação, caso mãe e bebê recebam mais tempo de cuidado e apoio ao AM, de maneira a persistir na amamentação após a alta. Reforça-se a importância organizacional dos serviços para a atenção e apoio à amamentação, que devem ser dados também ao RN pré-termo tardio internado, mas especialmente aos bebês gravemente doentes admitidos em unidades neonatais $^{(9)}$.

No presente estudo, esperava-se uma prevalência do $A M E$ mais elevada no $H F$, por possuir uma característica de população menos grave e mais desenvolvida socialmente, entretanto, encontrou-se o contrário, embora essa diferença não tenha sido estatisticamente significante. Considerando as diferenças entre os grupos, o tamanho amostral pequeno e consequente- mente alguns intervalos de confiança alargados, os fatores associados não conseguem por si só explicar tal fato.

Corroborando com alguns autores que afirmam que o IHAC convencional não é suficiente para apoiar o AM nessa clientela específica ${ }^{(5)}$, uma análise das diferenças na assistência dessas duas instituições pode complementar a análise das prevalências encontradas.

Pesquisadores de diferentes países têm proposto a expansão do IHAC convencional para outros cenários de cuidado, levando em consideração o contexto das unidades neonatais, as necessidades peculiares dos prematuros e/ ou RN gravemente doentes e a necessidade de um cuidado diferenciado para oferecer o apoio necessário à amamentação para essa população ${ }^{(5)}$. Essa proposta de expansão para unidades neonatais, denominado IHAC-Neo, baseia-se em três princípios norteadores para fundamentar as ações de apoio: foco e resposta às necessidades individuais de cada família, ações baseadas no cuidado centrado na família, e a continuidade do cuidado entre os períodos pré, peri e pós-natal, bem como após a alta hospitalar ${ }^{(5)}$.

A expansão dos Dez Passos do IHAC-Neo diferencia-se essencialmente pelo contexto das unidades neonatais, sendo que cinco deles foram estruturados diferentemente: passos 3, 4, 5, 8 e 9 . Nesta nova proposta, o passo 3 recomenda informar todas as gestantes hospitalizadas com risco de nascimento prematuro ou criança doente sobre o manejo da lactação e amamentação. O apoio precoce, sistemático e contínuo para as mães iniciarem a expressão láctea, principalmente quando os bebês não estão aptos a fazê-lo, são essenciais para superar as dificuldades fisiológicas e emocionais relacionadas ao amamentar no contexto da unidade neonatal(13).

O passo 4 propõe encorajar precocemente o contato pele a pele mãe-bebê de maneira contínua e prolongada (Cuidado Canguru). Con- 
siderando que nem sempre é possível realizar esse o contato ainda na sala de parto, dada a gravidade do RN naquele momento, há evidências de que o Cuidado Canguru precoce, contínuo e prolongado na unidade neonatal se constitui como intervenção eficaz na promoção do AM, produzindo prevalências geralmente mais altas que o cuidado convencional. O contato pele a pele estimula a produção láctea, aumenta a duração da amamentação, proporciona maior ganho de peso, diminui o tempo de hospitalização e favorece o vínculo afetivo ${ }^{(15)}$.

O passo 5 postula demonstrar às mães como iniciar e manter a lactação e estabelecer a estabilidade do bebê como único critério para o início precoce da amamentação(5).

O passo 8 defende o encorajamento da livre demanda ou, quando necessário, a semi-demanda de amamentação como uma estratégia transicional para prematuros ou bebês doentes. Maneiras diversificadas são empregadas para essa transição, dada as divergências e dificuldades desse manejo na prática ${ }^{(5)}$.

O passo 9, por sua vez, admite o uso de métodos alternativos à mamadeira pelo menos até o completo estabelecimento da amamentação e somente uso de bicos ou chupetas por motivos justificados. A utilização de protetores de mamilos e de chupeta confere um risco aumentado de 2 a 3 vezes de interrupção do AME na alta ${ }^{(13)}$.

Nesta perspectiva, cabe descrever e analisar algumas práticas diferenciadas encontradas à luz dessas recomendações, uma vez que as duas instituições em estudo são hospitais com título de Amigo da Criança e, teoricamente, já deveriam cumprir pelo menos os Dez Passos propostos pelo IHAC convencional.

Com a implantação da residência de enfermagem na unidade neonatal do HU, foi possível organizar uma assistência sistematizada, individualizada e diferenciada para os RN prematuros e suas famílias, auxiliando e apoiando o AME, por meio do programa "Uma Rede de Apoio à Família Prematura", criado desde 2007. Este projeto já vem aplicando os três princípios norteadores do IHAC-Neo(16). As atividades têm como referência o cuidado centrado na família e são desenvolvidas desde o nascimento, durante todo o processo de hospitalização, no domicílio e no ambulatório após a alta hospitalar, com a finalidade de atender e apoiar as necessidades das famílias de prematuros nascidos no HU. Além disso, dispõe da atuação do Banco de Leite Humano no apoio às mães dos prematuros para ordenha mamária e manutenção da produção láctea.

No HF, apesar de contar com uma equipe multiprofissional treinada para promover e apoiar o AM durante o período de internação, o acompanhamento do prematuro após a alta não está organizado sistematicamente em ambulatório de seguimento especializado e se dá na procura direta da família pelo posto de coleta/ sala de apoio à amamentação da maternidade da instituição e nas consultas com pediatra do convênio / particular ou nas Unidades Básicas de Saúde.

Após a alta hospitalar, é muito comum que as mães enfrentem obstáculos para continuar amamentando seu filho prematuro. É imprescindível que essas mães recebam apoio após a alta, uma vez que a transição para a casa exige adaptação que costuma ser considerada um desafio, mesmo se a amamentação estiver bem estabelecida ${ }^{(16)}$. Recomenda-se a atuação dos profissionais de saúde que atenderam diretamente os prematuros e suas mães, pois estariam mais bem familiarizados para orientar as famílias, antecipar e resolver efetivamente as limitações da lactação em casa ${ }^{(15)}$. Isso acontece no HU, onde as famílias são adotadas por uma enfermeira residente a fim de propiciar maior vínculo, continuidade e melhor apoio para a família desde o nascimento até um ano de idade. 
Observou-se, portanto, que ações especializadas para o apoio e incentivo do início precoce do AM durante a internação e o seguimento após alta hospitalar para o favorecimento e a manutenção do AME do prematuro estão sistematicamente implantadas no HU, o que pode ocorrer no HF, embora não sistematicamente, nem necessariamente para todas as famílias.

Em acordo com o IHAC-Neo, recomenda-se práticas que precisam ser incorporadas em ambas instituições, como: aumentar a permanência das mães nas unidades neonatais sem restrições de horário; favorecer o contato precoce pele a pele entre a mãe e o RN prematuro de forma contínua e prolongada; favorecer o conhecimento da gestante de risco ao parto prematuro sobre o AM do prematuro; não fixar horários para as mamadas em seio materno; incentivar AM de livre demanda e semi-demanda; apoiar o início e manter a lactação, instituindo a estabilidade clínica do bebê como único critério para o início precoce da sucção; utilizar métodos alternativos como a mamadeira, protetores de mamilos e chupetas somente com motivos justificados; incluir e preparar melhor os pais para a continuidade do AM; e assegurar o acompanhamento pós-alta por uma equipe treinada para minimizar a insegurança desse período e favorecer a continuidade do AME.

Vale lembrar que os prematuros tardios, embora nem sempre fiquem internados prolongadamente, também exigem mais atenção que os nascidos a termo para alcançar o AME e sua manutenção. Portanto, a família precisa receber mais apoio e ser preparada de maneira diferenciada durante a internação, com grandes esforços para o alojamento conjunto, além de prover acompanhamento especializado precoce após a alta.

A estratégia IHAC-Neo propõe esse conjunto de ações para essa população vulnerável a fim de avançar nas dificuldades e concretizar a prática do AM em bebês prematuros.

\section{CONCLUSÃO}

Os dados obtidos neste estudo permitiram concluir que houve baixa prevalência do AME no momento da alta e 15 dias após a alta nos dois hospitais em estudo.

Os fatores associados com a interrupção do AME na alta foram: tipo de parto, sexo do RN e uso de oxigenoterapia, no HU; e história de parto prematuro, intercorrência no parto, reanimação em sala de parto, Apgar menor que 7 no $5^{\circ} \mathrm{mi}-$ nuto e uso de oxigênio, no HF.

Logo após a alta hospitalar, as variáveis associadas com a descontinuidade do AME no HU foram: tipo de gestação, uso de oxigenoterapia e trauma mamilar. No HF, por sua vez, estiveram associados: companheiro, escolaridade da mãe, história de parto prematuro, intercorrência no parto, Apgar menor que 7 no $5^{\circ}$ minuto, reanimação na sala de parto, IG ao nascer, uso de oxigenoterapia e trauma mamilar.

Apesar das associações encontradas, concluiu-se também que o cumprimento dos passos do IHAC convencional não é suficiente para atender a essa população vulnerável e outras ações específicas podem interferir no processo do AM do prematuro.

\section{REFERÊNCIAS}

1. Eidelman Al, Schanler RJ. Section on Breastfeeding Executive Committee. Breastfeeding and the use of human milk: policy statement. Pediatrics. 2012;129:e827---41.

2. Brasil. Ministério da Saúde. Secretaria de Atenção à Saúde. Departamento de Atenção Básica. Saúde da criança: nutrição infantil: aleitamento materno e alimentação complementar / Minis- 
tério da Saúde, Secretaria de Atenção à Saúde, Departamento de Atenção Básica. Brasília (DF): Ministério da Saúde; 2009.

3. Cruz MCC, Almeida JAG, Engstrom EM. Práticas alimentares no primeiro ano de vida de filhos de adolescentes. Rev Nutr. 2010; 23(2): 201-210.

4. Rollins NC, Bhandari N, Hajeebhoy N, Horton S, Lutter CK, Martines JC, et al. Why invest, and what it will take to improve breastfeeding practices? Lancet. 2016; 387: 491-504.

5. Nyqvist HK, Häggkvist AP, Hansen MN, Kylberg $E$, Frandsen $A L$, Maastrup $R$, et al. Expansion of the ten steps to successful breastfeeding into neonatal intensive care: expert group recommendations for three guiding principles. J Hum Lact. 2012; 28(3):289-96.

6. Rodrigues AP, Martins EL, Trojahn TC, Padoin SMM , Paula CC, Tronco CS. Manutenção do aleitamento materno de recém-nascidos pré-termo: revisão integrativa da literatura. Revista Eletrônica de Enfermagem. 2013; 15(1): 253-64.

7. Sassá AH, Schmidt KT, Rodrigues BC, Ichisato SMT, Higarashi IH, Marcon SS. Bebês pré-termo: aleitamento materno e evolução ponderal. Rev. bras. enferm. 2014; 67(4): 594-600.

8. Silva WF, Guedes ZCF. Tempo de aleitamento materno exclusivo em recém-nascidos prematuros e a termo. Rev CEFAC. 2013; 15(1): 160-171.

9. Silva L, Elles M, Silva M, Santos I, Souza K, CarvaIho S. Social factors that influence breastfeeding in preterm infants: a descriptive study. Online Brazilian Journal of Nursing [serial on the Internet]. 2012; 11(1). [Cited 2017 Oct 08]. Available from: http://www.objnursing.uff.br/index.php/ nursing/article/view/3528

10. Costa BC, Vecchi AA, Granzotto JA, Lorea CF, Mota DM, Albernaz EP; et al. Análise comparativa de complicações do recém-nascido prematuro tardio em relação ao recém-nascido a termo. Boletim Científico de Pediatria. 2015; 4(2): 33-37.

11. Briere CE, Lucas R, McGrath JM, Lussier M, Brownell $E$. Establishing breastfeeding with the late preterm infant in the NICU. J Obstet Gynecol Neonatal Nurs. 2015; 44(1):102-13.

12. Machado LC Jr, Passini R Jr, Rosa IR. Late prematurity: a systematic review. J Pediatr (Rio J). 2014; 90(2): 21-31.
13. Maastrup R, Hansen BM, Kronborg H, Bojesen $S N$, Hallum K, Frandsen A; et al. Factors associated with exclusive breastfeeding of preterm infants. Results from a prospective national cohort study. PLoS ONE. 2014; 9(2): e89077.

14. Prior E, Santhakumaran S, Gale C, Philipps LH, Modi N, Hyde MJ. Breastfeeding after cesarean delivery: a systematic review and meta-analysis of world literature. Am J Clin Nutr. 2012; 95(5): 1113-35.

15. Briere CE, McGrath J, Cong X, Cusson R. An Integrative Review of factors that influence breastfeeding duration for premature infants after NICU hospitalization. JOGNN. 2014; 43: 272-281.

16. Bengozi TM, Souza SNDH, Rossetto EG, Radigonda B, Hayakawa LM, Ramalho DP. Uma rede de apoio à família do prematuro. Cienc Cuid Saude. 2010; 9(1): 155-160.

Todos os autores participaram das fases dessa publicação em uma ou mais etapas a seguir, de acordo com as recomendações do International Committe of Medical Journal Editors (ICMJE, 2013): (a) participação substancial na concepção ou confecção do manuscrito ou da coleta, análise ou interpretação dos dados; (b) elaboração do trabalho ou realização de revisão crítica do conteúdo intelectual; (c) aprovação da versão submetida. Todos os autores declaram para os devidos fins que são de suas responsabilidades o conteúdo relacionado a todos os aspectos do manuscrito submetido ao OBJN. Garantem que as questões relacionadas com a exatidão ou integridade de qualquer parte do artigo foram devidamente investigadas e resolvidas. Eximindo, portanto o OBJN de qualquer participação solidária em eventuais imbróglios sobre a matéria em apreço. Todos os autores declaram que não possuem conflito de interesses, seja de ordem financeira ou de relacionamento, que influencie a redação e/ou interpretação dos achados. Essa declaração foi assinada digitalmente por todos os autores conforme recomendação do ICMJE, cujo modelo está disponível em http://www. objnursing.uff.br/normas/DUDE_final_13-06-2013.pdf

Recebido: 07/08/2016

Revisado: 03/10/2017

Aprovado: 03/10/2017 